\title{
An Investigation of Various Segmentation Methods Used in Iris recognition System
}

\author{
Tanni Dhoom \\ Lecturer \\ Premier University \\ Chittagong, Bangladesh
}

\author{
Munmun Biswas \\ Lecturer \\ BGC Trust University Bangladesh, \\ Chittagong, Bangladesh
}

\begin{abstract}
A biometric framework offers automatic identification of a human being in view of the special feature or characteristic which is being controlled by the individual. The iris, one of the biometrics emerges among other biometric strategies due to its unique features like stability and accuracy. Iris Recognition has its significant applications in the field of surveillance, forensics and furthermore in security purposes As of late, iris recognition is produced to a few dynamic areas of research, for example, Image Acquisition, restoration, quality assessment, image compression, Image segmentation, noise reduction, normalization, feature extraction, iris code matching, looking vast database, execution under shifting condition and multi bio-metrics. This paper reviews a foundation of iris recognition and literature of late proposed strategies in various fields of iris recognition system.
\end{abstract}

\section{General Terms}

Image processing, Pattern recognition, Iris recognition

\section{Keywords}

Biometric, Iris Segmentation, Feature extraction

\section{INTRODUCTION}

Iris recognition is a biometric recognition system refers to image processing and furthermore pattern recognition area. The expression "Biometrics" refers to a science including the measurable examination of biological characteristics. A biometric template can give a powerful, standardized and extremely separating feature's by using distinctive physical (e.g. hand profile, palm vein, fingerprints, DNA testing, face, iris, palm print, retinal scanning, and so on) and conduct (e.g. walk estimation, voice, keystroke and signature) characteristics of each which will hence be in correlation with various formats to find out identity in security reason.

These days security is one of the critical factors in the field of information, business, and online business, military and so forth. Consequently personal identification has turned into a critical topic [5]. Iris deals in identification of individual in light of their physiological attributes. Moreover the quality, all-inclusiveness, permanence, collect-ability and novel data estimated in a solitary iris are considerably more noteworthy than other biometric data [24]. Utilization of iris biometrics innovation include: identification cards and passports, border control and other government programs, jail security, database access and PC login, schools, aeronautics security, clinic security, controlling access to confined regions, going into to structures and houses[5]. The United Nations High Commissioner for Refugees (UNHCR) utilized iris acknowledgment for Afghan outcasts [27]. Iris acknowledgment is utilized in prisons for the acknowledgment of detainees. Air terminals in different countries utilize iris recognition at their visitors and immigration control [5]. Iris recognition perceives individuals by using the particular iris pattern information and standing out it from database's reference. For instance, it is stable during a person's lifetime and it can't change carefully. Its exactness rate is higher in correlation with various biometric recognition approaches like voice recognition, fingerprint recognition, face recognition and so on [29],[30],[31]. As surface of iris pattern does not have any connections with a person's genetic structure and in light of the fact that it is made by dislocated procedures, it can work in verification and identification modes [7], [28]. Because of the little size of the iris image, a database of iris image of a vast population can be saved on a personal computer or flash memory stick and carried [7].Among all biometrics, the iris biometric is the most remarkable and vigorous biometric - even the iris patterns of twins are different[7].

\section{IRIS RECOGNITION SYSTEM}

Iris is the portion of the human eye. The human eye as appeared in Fig 1 is just about a spherical ball with a slight lump in the front part. Iris, is the best biometric attribute than different characteristics as these get modified with the age and infections however iris pattern remains unaltered for the duration of the life. Iris is gaining lot of attention of consideration because of its performance and quantifiable. Situated among cornea and eye lens, iris is inside ensured yet externally visible.

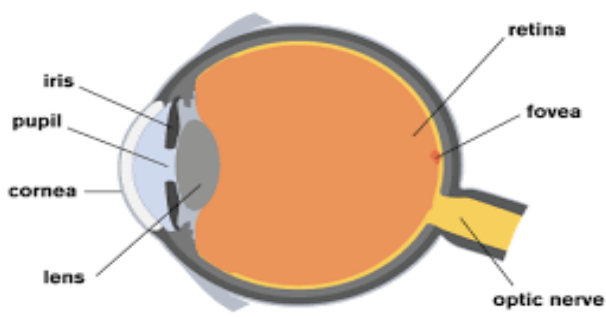

Fig 1: The human eye

The iris comprises of particular characteristics such as the freckles, coronas, strips, furrows and so on. Extensive Research throughout the decade has expanded the reputation of iris. The issues with iris distinguishing reside in the structure of organ itself. Iris images caught are blocked by eyelids and eyelashes. The structure of iris and pupil isn't round and concentric. The iris boundaries are attempted to be circular, which prompts inappropriate localization of iris. Hence, powerful localization and normalization strategies are basic.

There are five phases of iris recognition; acquisition, segmentation, normalization, feature extraction and recognition. Each stage applies distinctive procedures. Iris 
segmentation refers to the way toward extracting region that provides information of iris pattern [40], [42]. It is the way toward finding the inner and outer boundaries of iris. Normalization refers to the getting ready of upgraded iris image. It changes over the Cartesian directions to polar directions. The following stage is feature extraction, to extract features from standardized iris image and making unique iris codes [43]. The iris codes are additionally utilized for correlations. This article emphasize on these four phases and the recognition rate to demonstrate the precise execution. The parameters which measure the execution are False Acceptance Rate and False Rejection Rate. The FAR is the recurrence that a non-approved in person is accepted as authorized. FRR is the recurrence that an approved individual is rejected access. The overview of iris recognition is appeared in Fig. 2.

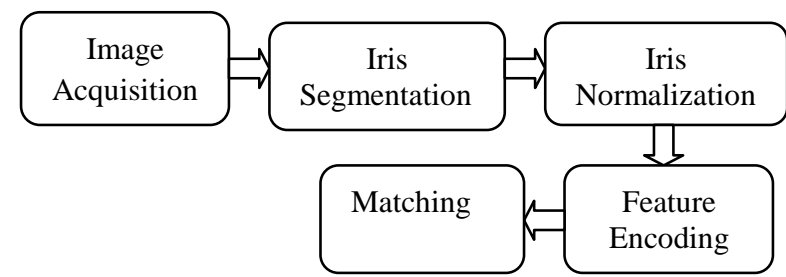

Fig 2: Iris recognition System Overview

\section{IMAGE ACQUISITION \& PREPROCESSING}

Taking a photo from iris is the underlying phase of an irisbased recognition system. Accomplishment of other recognition stages is dependent on the nature of the images taken from iris during image acquisition stage. Images accessible in CASIA database need appearance in pupil and iris areas since infrared was utilized for imaging. Moreover, if obvious light is utilized during imaging for those people whose iris is dark, a slight differentiation comes to presence among iris and pupil which makes it difficult to isolate these two areas [2]. A procured iris image contains some 'unuseful' parts as well (e.g. eyelid, pupil and so on.). In this way, coordinate utilization of the image isn't conceivable. Additionally an adjustment out distance from camera-to-face may result in variety sizes of same iris. Besides, the brightness isn't consistently conveyed a direct result of non-uniform illumination. Along these lines, preprocessing of original image is expected to localize, normalize iris, and reduce the impact of malefactors [11]. In normalization stage, an approach in view of Daugman's method is utilized. In this way, iris area is gotten as a normalized strip as to iris limits and pupillary center [2]. The normalized iris image still has low difference and may have non-uniform illumination caused by the situation of light sources. All these may influence subsequent feature extraction and pattern matching. So, the iris image ought to be enhanced by methods for histogram equalization and remove noise by filtering the image [11].

\section{DISTINCTIVE APPROACHES OF SEGMENTATION}

There are fundamental four essential strategies utilizes in Irish segmentation. They can be utilized remarkably or in combination of them. They are Hough Transform, Canny Edge Detection, Intigro differential Operator and Circular Hough Transformation.

\subsection{Hough Transformation}

The Hough Transform is an algorithm displayed by Paul Hough in 1962 for the recognition of features of a specific shape like lines or circles in digitalized images. The classic
Hough Transform is a standard algorithm for line and circle discovery. It very well may be connected to numerous computer vision issues as most images contain feature boundaries which can be depicted by regular curves. The principle favorable position of the Hough transform technique is that it is tolerant to gaps in feature boundary descriptions and is generally unaffected by image noise, dissimilar to edge detectors. [3]

\subsection{Canny edge Detection}

The Canny Edge detection method is used to discover the iris and pupil boundaries from the caught image. This gives the effective edges of eye. So we can get the correct pupil edge to recognize the image. The algorithm keeps running in 5 separate steps: They are Smoothing, Finding gradients, Nonmaximum suppression, Double thresholding, Edge following by hysteresis [25]. The primary point of smoothing is to remove the noise from the obscure images. At the point when the grayscale intensity of the image is changed to discover the edges essentially canny algorithm is utilized. Those regions are found by deciding gradients of that image. From the smoothed images the gradient points are decides every pixel.

It is to change over the obscured edges in the image of the gradient magnitude to make sharp edges. Fundamentally this is finished by preserving all neighborhood maxima in the gradient image, and erasing everything else. The edge-pixels staying after the non-maximum suppression step are set apart with their quality pixel-by-pixel. Strong edges are translated as "specific edges", and can quickly be incorporated into the last edge image. Weak edges are incorporated if and only if they are associated with strong edges. Edge tracking can be executed by BLOB-investigation (Binary Large Object).

\subsection{Integro-Differential Operator}

This approach is looked like as a standout amongst the most referred to approaches in the overview of iris recognition. John Daugman first proposed the model of an integro differential administrator for sectioning the iris in the middle of 1992 to 1994[5]. It finds both internal and the external boundaries of the iris region. The external and additionally the internal boundaries are alluded to as limbic and pupil boundaries. The parameters for example, center and radius of the circular boundaries are being looked in the three dimensional parametric space keeping in mind the end goal to amplify the evaluation functions engaged with the model. It was really in view of the way that the illumination contrasts amongst inside and outside of pixels in iris edge circle is most extreme and it acts as circular edge detector. This algorithm accomplishes high performance in iris recognition. It is having a drawback that it experiences substantial computation [3].

\subsection{Circular Hough transformation}

Circular Hough Transformation is really an adjusted variant of Hough transform. In this strategy Hough transform joins with canny edge detector. Richard Duda and Peter Hart expands the

General Hough Transform in1972 as Circular Hough Transform (CHT), is utilized to detect circles. The In Generalized Hough Transform. edge distinguished from the Canny edge detector forms the contribution to extricate the circle utilizing the Circular Hough Transform[9].The general Hough transform can be utilized to identify geometric shapes that can be composed in parametric shape for example lines, circles, parabolas, and hyperbolas. The circular Hough 
transform can be utilized to identify the circles of a known radius in an image.

The transform is processed by drawing circles of a given radius at each point in the edge image. For each point where the perimeter of a drawn circle passes, the coordinate was augmented by 1 . This was improved the situation each circle drawn to make an accumulation array. A circle is shown by peaks in the accumulation array (Hough space) [3].Detection of circle utilizing this transformation requires learning of the radius. [10]

Table 1 gives the comparison of algorithms used in different segmentation method:

\begin{tabular}{|c|c|c|c|}
\hline Segmentation Method & Features & Matching process & Result \\
\hline $\begin{array}{l}\text { Image intensity gradient } \\
\text { and Hough transform }\end{array}$ & $\begin{array}{l}\text { Laplacian pyramid to represent } \\
\text { the spatial characteristics of iris } \\
\text { image. }\end{array}$ & $\begin{array}{l}\text { Normalized } \\
\text { correlation }\end{array}$ & $\begin{array}{l}\text { Matching process is tedious. It might be } \\
\text { appropriate for identification stage not for } \\
\text { recognition, not reasonable for usage. }\end{array}$ \\
\hline Circular iris shape & Zero crossing and 1D signals & $\begin{array}{l}\text { Two dissimilarity } \\
\text { functions: the } \\
\text { learning and the } \\
\text { classification }\end{array}$ & $\begin{array}{l}\text { Generally low recognition rate, speedier } \\
\text { matching process yet high EER, } \\
\text { straightforward } 1 \mathrm{D} \text { feature vector. }\end{array}$ \\
\hline $\begin{array}{l}\text { Gray level information, } \\
\text { Canny edge detection } \\
\text { and Hough transform } \\
\end{array}$ & $\begin{array}{l}\text { 1D real-valued feature vector } \\
\text { using multichannel spatial filters } \\
\text { with the length of } 384\end{array}$ & Nearest feature line & $\begin{array}{l}\text { Generally moderate component extraction } \\
\text { process. }\end{array}$ \\
\hline $\begin{array}{l}\text { Gray level information, } \\
\text { Canny edge detection } \\
\text { and Hough transform }\end{array}$ & $\begin{array}{l}\text { 1D real-valued feature } \\
\text { vector using Dyadic wavelet with } \\
\text { the length of } 160\end{array}$ & $\begin{array}{l}\text { Weighted } \\
\text { Euclidean distance }\end{array}$ & local features are utilized for recognition \\
\hline Hough transform & $\begin{array}{l}\text { Direct linear discriminate analysis } \\
\text { (DLDA) }\end{array}$ & Hamming distance & Hough transform \\
\hline $\begin{array}{l}\text { Integro-differential } \\
\text { operator and angular } \\
\text { deformation model }\end{array}$ & $\begin{array}{l}\text { Independent component } \\
\text { Analysis and Biorthogonal } \\
\text { wavelets }\end{array}$ & Hamming distance & Enhanced execution on non-perfect dataset \\
\hline Hough transform & Patch coding & Hamming distance & $\begin{array}{l}\text { Moderately lower recognition rate on } \\
\text { complex dataset }\end{array}$ \\
\hline Hough transform & $\begin{array}{l}\text { Zero crossings of 1D Discrete } \\
\text { Cosine Transform (DCT) }\end{array}$ & Hamming distance & $\begin{array}{l}\text { Faster element extraction process, Higher } \\
\text { recognition rates and lower EER }\end{array}$ \\
\hline $\begin{array}{l}\text { Log and canny edge } \\
\text { detector }\end{array}$ & $\begin{array}{l}2 \mathrm{~d} \text { gabor wavelets and } \\
\text { Biorthogonal wavelet }\end{array}$ & $\begin{array}{l}\text { Intra and inter-class } \\
\text { hamming distance }\end{array}$ & $\begin{array}{l}\text { Accuracy rate is so high. FAR and FRR is } \\
\text { so low. }\end{array}$ \\
\hline $\begin{array}{l}\text { Canny edge and Circular } \\
\text { Hough Transformation }\end{array}$ & Biorthogonal wavelet & $\begin{array}{l}\text { Intra and inner-class } \\
\text { hamming distance }\end{array}$ & $\begin{array}{l}\text { Angular deflections influence then } \\
\text { recognition execution of the system. In } \\
\text { spite of the fact that Higher recognition } \\
\text { rate. }\end{array}$ \\
\hline $\begin{array}{l}\text { Canny edge and Circular } \\
\text { Hough Transformation }\end{array}$ & $\begin{array}{l}\text { 2d gabor filter and wavelet } \\
\text { transformation }\end{array}$ & Hamming distance & $\begin{array}{l}\text { Precision rate is high. FAR and FRR is } \\
\text { moderately so low, Multistage recognition. }\end{array}$ \\
\hline $\begin{array}{l}\text { Circular Hough } \\
\text { Transform, Canny, Sobel } \\
\text { and Prewitt edge } \\
\text { detection }\end{array}$ & $\begin{array}{l}\text { Feature vector from wavelet } \\
\text { transformation and } 1 \mathrm{~d} \text { gabor filter }\end{array}$ & Hamming distance & $\begin{array}{l}\text { Sobel edge detector demonstrates great } \\
\text { execution in accuracy. }\end{array}$ \\
\hline $\begin{array}{l}\text { Canny edge and Circular } \\
\text { Hough Transformation }\end{array}$ & $\begin{array}{l}\text { Binary coded Feature vector from } \\
\text { Haar wavelet transform }\end{array}$ & Hamming Distance & $\begin{array}{l}\text { Very straightforward and furthermore } \\
\text { successful yet requires stable equipment. }\end{array}$ \\
\hline $\begin{array}{l}\text { Integro-differential } \\
\text { operator }\end{array}$ & $\begin{array}{l}\text { Binary features vector using 2D } \\
\text { Gabor filters. }\end{array}$ & Hamming distance & $\begin{array}{l}\text { Good recognition rate and gives a speedier } \\
\text { iris/pupil detection process on perfect iris } \\
\text { images, not working on non-perfect } \\
\text { images. }\end{array}$ \\
\hline $\begin{array}{l}\text { Active contours and } \\
\text { generalized coordinates }\end{array}$ & Iris Code & Hamming distance & $\begin{array}{l}\text { Gaze deviation has been evaluated, low } \\
\text { time complexity. }\end{array}$ \\
\hline
\end{tabular}

\section{FEATURE EXTRACTION AND ENCODING}

After image segmentation process features will be observed from the image pixel values followed by encoding process to get information that is needed for matching process. Various feature outcomes is possible to get from different segmentation process

\subsection{IrisCode}

IrisCode is based Daugman's calculation. This is these days the most utilized algorithm in commercial devices, on account of its speed of matching with low false match rates. With
IrisCode the obvious characteristics of the iris are transformed into a phase sequence, which contains data on the introduction, spatial frequency and position of segments in the iris. The phase isn't influenced by differentiate, camera gain or illumination levels. The consequence of the algorithm is an IrisCode, 256 bytes of information which describe the phase qualities of the iris in a polar coordinate system. Utilizing 256 byte formats for all scanned irises permits matching up to 500.000 templates for each second utilizing Iris Code [38].

\subsection{Biorthogonal wavelet}

A biorthogonal wavelet is where the related wavelet transform is invertible however not really orthogonal. Planning 
biorthogonal wavelets permits a bigger number of degrees of flexibility than orthogonal wavelets. One extra level of flexibility is the likelihood to develop symmetric wavelet functions [32].

\subsection{Gabor wavelets}

Gabor filtering is a well-known technique in texture analysis. It cannot just concentrate useful information in particular band pass channels yet in addition decompose this information into biorthogonal segments as far as spatial frequencies. The Gabor space is very useful in image processing applications [11]. A feature vector is an accumulation of the considerable number of features from each filtered sub image. The average absolute deviation of each filtered image comprises the segments of our feature vector. These features are arranged to shape a 1D feature vector of length 160 for each input image [11].The dimensions of the feature vector extracted from iris area have to be as small as possible. Regarding high dimensions of the image drawn, Wavelet transform was performed in order to decrease the dimensions in the way that important information existing in tissue can be preserved in spite of downsizing image dimensions [2].

\subsection{Haar wavelet}

The Haar wavelet is the simplest wavelet transform. Haar wavelets are identified with a mathematical operation called Haar transform, which fills in as a model for all other wavelet transforms. The Haar transform breaks down a discrete signal into two sub signals of a large portion of its length. One sub signal is a running average or trend, the other sub signal is a running distinction or fluctuation. During computation, the breaking down wavelet is moved over the full space of the investigated work. Haar wavelet transform is performed by computing the running averages and contrasts by means of scalar items with scaling signs and wavelets [32]

\subsection{Laplacian Pyramid}

The Laplacian Pyramid decomposition initially created by Burt and Adelson. Development of the Laplacian Pyramid starts first with convolution of the Iris Image with LoG Mask, in order to yield low-pass Gaussian filtered images. The Laplacian is then figured as the contrast between the original picture and the low pass separated image. The development is joined by up sampling and interpolation. Up sampling is accomplished by the insertion of zeros between each row and column of the down examined Gaussian image. The Mask is utilized as an interpolation filter and the factor 4 is fundamental since $3 / 4$ pixels are recently embedded zeros [54]

\subsection{Direct linear discriminate analysis (DLDA)}

Linear Discriminate Analysis is an outstanding plan for feature extraction and measurement decrease. It has been utilized generally in numerous applications, face recognition, image retrieval, microarray data classification, and so forth. Traditional LDA projects the information onto a lowerdimensional vector space to such an extent that the ratio of the between-class separations to the within-class distance is expanded, along these lines accomplishing maximum discrimination. The optimal projection (transformation) can be promptly computed by applying the Eigen decomposition on the scatter matrices. A natural constraint of traditional LDA is that its target work requires the no singularity of one of the scatter matrices. For some applications for example face recognition, all scatter matrices being referred to can be singular since the information is from a high-dimensional space, and in general, the measurement surpasses the number of data points [21]

\subsection{Patch coding}

The 'patch' is the basic fragment used in this method. To obtain optimum performance it is needed to tune the length, width, orientation (angle) and the relative position of a series of patches. The procedure for coding a patch is shown in Figure 3.

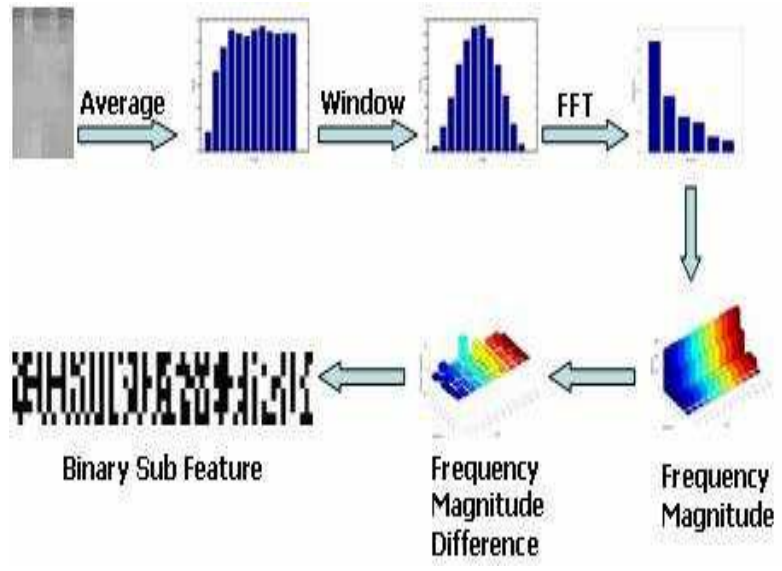

Fig 3: Procedure of patch coding

First a 1D intensity signal is obtained by averaging the patch across its width to reduce noise. Using broad patches also makes iris image registration easier, which is important for rotation invariant iris recognition. The FFT is then applied to this 1D signal to obtain spectral coefficients.

In order to reduce the spectral leakage during the FFT, a window is employed before the FFT. The Frequency Magnitude Differences between adjacent patches are calculated and a short binary code is generated from the zero crossings of each difference. These constitute the feature vectors of our iris code [53].

\subsection{Feature Vector by Zero Crossing}

At the point when a signal incorporates important structures that have a place with various scales, usually helpful to reorganize the signal information into a set of "detail components" of varying size. It is realized that one can get the position of multiscale sharp varieties focuses from the zero crossing of the signal convolved with the laplacian of a gaussian [33]. This methodology has been utilized in many pattern recognition applications. An essential issue is to understand whether the zero-crossing characterize an entire and stable portrayal of the original signal. Surely for pattern recognition applications, we remove some critical parts of the signal, while representing to it with multiscale zero-crossing. Zero crossing of are assessed from sign changes of its samples. The situation of each zero crossing is assessed with a linear interpolation between two samples of various sign.

In this way if Iris Signature has $\mathrm{N}$ nonzero samples, since there are at most $\operatorname{Nog}(\mathrm{N})$ samples in its discrete wavelet representation, the number of operations to get the situation of the zero crossing is $\mathrm{O}(\mathrm{N} \log (\mathrm{N}))$ It is avoided the coarsest level keeping in mind the end goal to get a robust representation in a noisy environment and diminishes the number of computations required, since information at fine goals levels is strongly influenced by noise and quantization errors which are because of the utilization of a rectangular grid in digital images[33]. Accordingly, to diminish such effects for the 
zero-crossing representation a few low resolution levels, excluding the coarsest one, will be utilized.

\section{MATCHING PROCESSES}

In this phase, there are various ways used in matching process. After feature extraction it is needed to match the features of the examined image with the learned dataset. By this survey, we get that the most common way is to use Hamming distance between the features. But there are some other processes used too.

\subsection{Hamming distance}

It is the easier and efficient among all the methods used in matching process. To calculate hamming distance we need Iris Codes which can be getting from segmentation and feature extraction process. The difference between input iris codes is called the Hamming Distance. If no less than one third of the iriscodes differs from each other, then it concludes that there is no statistical significant difference in between the IrisCodes and they are considered to be matched [38].

\subsection{Weighted Euclidian Distance}

For simplicity, iris matching is based on computing the Weighted Euclidean distance (WED) between the corresponding feature vectors. WED is defined in the following equation-

$$
W E D(k)=\sqrt{\sum_{i=1}^{B N} A_{i} \sum_{j=1}^{N}\left(f_{(i, j)}^{k}-f_{(i, j)}\right)^{2}}
$$

Where, $A_{i}$ is the ith weighting coefficient, BN is the quantity of sub images and $\mathrm{N}$ is the total number of features extracted from each sub image, $f_{(i, j)}$ and $f_{(i, j)}^{k}$ signify the jth feature of the ith sub image of the input iris image and that of iris listed by k. Here, we set the weighting coefficients I A for various feature sets separated from various sub images. These coefficients are resolved utilizing the closest neighbor classifier in algorithm. Features extracted from input iris are contrasted with irises in database. If the weighted Euclidean distance is least at $\mathrm{k}$ and not as much as a reasonable threshold then the input iris is distinguished as iris ordered by $\mathrm{k}$ [11].

\subsection{Normalized Correlation}

The circular pattern of iris speaks to in rectangular form is called Normalization. The distortion caused by pupil development can be diminished by Normalization. Normalization has done by utilizing Daugamn rubber sheet model [13]. In iris recognition, one of the most helpful matching is by corresponding the two normalized iris where connection is a single number that depicts the level of relationship between two variables.

\subsection{Nearest Feature lines}

The Nearest Feature Line (NFL) strategy expands the classification ability of the nearest neighbor (NN) technique by taking advantages of multiple (more than one) templates per class. It effectively enhances the classification performance particularly when the number of templates (sample size) per class is little; an issue regularly experienced in numerous applications, for example, in irish recognition issues. A critical part of pattern classification techniques is the meaning of a distance metric. Models incorporate Euclidean distance, Mahalanobis distance, Cosine distance, Hamming distance. Such distances are characterized between two feature points (FPs) which are vectors in a feature space. The nearest neighbor $(\mathrm{NN})$ order strategy ascertains the distance from the query FP to every template FP of a class, and chooses the minimum of those distances as the inquiry toclass distance. The nearest feature line (NFL) technique [55], initially proposed for face recognition and in this way utilized in numerous applications, takes favorable circumstances of multiple FPs per class to deduce intra-class variations from them and stretch out their ability to represent pattern classes [56].

\section{MULTIBIOMETRICS}

In iris recognition process needs a strong and clear database for learning and testing purpose. Here, different rich mutibiometrices are utilized in various experiments. In this review, we found that the most ordinarily utilized dataset is CASIA. It has 308 classes of irises and 2174 images (i.e. numerous images of 308 eyes). For each class of iris, three images are chosen arbitrarily as a training set, and the others utilized for testing [34].The iris images from UBIRISv1 [6] and some other databases is also used for the experimental analysis in some cases[8].

\section{CONCLUSION}

This paper gives writing survey of iris in a few dynamic zones of research, for example, image segmentation, noise reduction, normalization, feature extraction, feature encoding, iris code matching, condition and multibiometrics. Iris recognition is a reliable biometric system and as of now it is utilized in a few ongoing user applications, for example, ATM machines, prisoner authentication, banking, border controls, and airport and so on particularly in security reason to distinguish individuals.

\section{REFERENCES}

[1] A.V.G.S.Sastry, B. Durga Sri, "Enhanced Segmentation Method for Iris Recognition", International Journal of Computer Trends and Technology, volume-4, Issue-2, 2013, Pp 68-71,

[2] Mahmoud Mahlouji , Ali Noruzi , "Human Iris Segmentation for Iris Recognition in Unconstrained Environments", IJCSI International Journal of Computer Science Issues, Vol. 9, Issue 1, No 3, January 2012, pp $149-155$

[3] Nidhi Manchanda, Oves Khan, RishitaRehlan and JyotikaPruthi, "A Survey: Various Segmentation Approaches to Iris Recognition", International Journal of Information and Computation Technology, Vol. 3, N0. 5 (2013), pp. 419-424

[4] Sruthi.T.KLiterature review: Iris Segmentation Approaches for Iris Recognition Systems International Journal of Computational Engineering Research, Vol. 03, Issue 5, May 2013, pp 67-70

[5] ArezouBanitalebiDehkordi\& Syed A.R. Abu-Bakar , "A REVIEW OF IRIS RECOGNITION SYSTEM", JurnalTeknologi (Sciences \& Engineering), Vol. 77, Issue 1, 2015, pp275-282

[6] YachnaKumari, Mrs.Rohini Sharma, Iris Recognition System using Gabor Filter \& Edge Detection, International Journal on Recent and Innovation Trends in Computing and Communication, Volume: 2 Issue: 8, August 2014, pp $2265-2269$

[7] Neda Ahmadi, GholamrezaAkbarizadeh, "Iris Recognition System based on Canny and LoG Edge Detection Methods", Journal of Soft Computing and 
Decision Support Systems, Vol.2, No.4, August 2015: pp 26-30

[8] DeepikaPrashar, Mnupreet Kaur , "Human Eye Iris Recognition Using Discrete 2d Reverse Biorthogonal Wavelet 6.8", INTERNATIONAL JOURNAL OF SCIENTIFIC \& TECHNOLOGY RESEARCH VOLUME 3, ISSUE 8, AUGUST 2014, pp 266 -270

[9] Nitasha, Shammi Sharma, Reecha Sharma, "Comparison Between Circular Hough Transform And Modified Canny Edge Detection Algorithm For Circle Detection", International Journal of Engineering Research \& Technology (IJERT) Vol. 1 Issue 3, May - 2012, pp 1-5.

[10] R.B. Dubey , Abhimanyu Madan, “ Iris Localization using Daugman'sIntero-Differential Operator",International Journal of Computer Applications, Volume 93, No 3, May 2014, pp 6-12

[11] Li Ma, Yunhong Wang, Tieniu Tan, "Iris Recognition Based on Multichannel Gabor Filtering", The 5th Asian Conference on Computer Vision, Melbourne, Australia ,January 2002, pp 23--25.

[12] S.S. Kulkarni, G.H. Pandey, A.S.Pethkar, V.K. Soni, \&P.Rathod, "An Efficient Iris Recognition Using Correlation Method", International Journal of Information Retrieval, ISSN: 0974-6285 Vol. 2, Suppl. Issue 1, 2009, pp. 31-40

[13] Tai Sing Lee, "Image representation using 2D Gabor wavelet ", IEEE TRANSACTIONS ON PATTERN ANALYSIS AND MACHINE INTELLIGENCE, VOL. 18, NO. 10, OCTOBER 1996 pp. 959 - 971.

[14] K. C. Chandra sekaran , Dr. K. Kuppusamy , "Efficiency of Gaussian Pyramid Compression Technique for Biometric Images", IJCSI International Journal of Computer Science Issues, Vol. 11, Issue 3, No 1, May 2014, pp 77-82

[15] MakramNabti,* LahouariGhouti and Ahmed Bouridane, "An effective and fast iris recognition system based on a combined multiscale feature extraction technique", The journal of the pattern recognition society. vol. 41, 2008, pp 868-879.

[16] Vatsa, M., Singh, R., \& Gupta, P. (2004).” Comparison of iris recognition algorithms". International Conference on Intelligent Sensing and Information Processing, 2004. pp354-358.

[17] Vatsa, Mayank\& Singh, Richa\&Noore, Afzel. (2008). Improving Iris Recognition Performance Using Segmentation, Quality Enhancement, Match Score Fusion, and Indexing. IEEE Transactions on Systems, Man, and Cybernetics, Part B: Cybernetic. Vol. 38, pp. $1021-1035$

[18] Mriganawalia, Dr. Shaily Jain, "Iris Recognition System Using Circular Hough Transform", International Journal of Advance Research in Computer Science and Management Studies, Volume 3, Issue 7, July 2015, pp

[19] PROF. MANISHA MORE, PROF. VISHAK, "A Survey on Iris Recognition Techniques", International Journal of Novel Research in Computer Science and Software Engineering Vol. 2, Issue 1, pp: (89-94), Month: January - April 2015,
[20] Chengqiang Liu, MeiXie, "Iris Recognition Based on DLDA", The 18th International Conference on Pattern Recognition (ICPR'06), 2006, pp 21.

[21] Gafar Zen AlabdeenSalh, Abdelmajid Hassan Mansour, Elnazier Abdallah Mohammed, "Human Iris Recognition Using Linear Discriminant Analysis Algorithm", International Journal of Computer Applications Technology and Research ,Volume 4- Issue 5, pp. 395 404, 2015

[22] Hua Yu, Jie Yang A direct LDA algorithm for highdimensional data with application to face recognition, The Journal of The Pattern Recognition Society, Published by Elsevier Science Ltd. 2001, pp 2067- 2070

[23] Nivedita S. Sarode, A. M. Patil, "Review of Iris Recognition: An evolving Biometrics Identification Technology", International Journal of Innovative Science and Modern Engineering (IJISME),Volume-2 Issue-10, September 2014, pp 34-40

[24] Sonia Sangwan, Reena Rani A Review on: Iris Recognition, International Journal of Advanced Research in Mechanical Engineering \& Technology (IJARMET),Vol. 1, Issue 1 (Apr. - Jun. 2015) pp 45-47

[25] Chinni. Jayachandra, H.Venkateswara Reddy, “ Iris Recognition based on Pupil using Canny edge detection and K-Means Algorithm", International Journal Of Engineering And Computer Science, Volume 2,Issue 1 Jan 2013, pp 221-225

[26] D. M. Monro and Z. Zhang, "An effective human iris code with low complexity", IEEE International Conference on Image Processing 2005, Genova, 2005, pp. III-277.

[27] 2003. Iris Testing of Returning Afghans Passes 200,000 Mark. Available: http://www.unhcr.org/cgibin/texis/vtx/search?docid=3f86b4784 .

[28] Muron, A., Pospisil, J. (2000). The human iris structure and its usages. Acta Univ. Palacki. Olomuc. Fac. Rerum Nat. Phys, Vol. 39, pp 87-95.

[29] Daugman, J. G. (1993). High confidence visual recognition of persons by a test of statistical independence. Pattern Analysis and Machine Intelligence, IEEE Transactions on, Vol.15 issue11, $1148-1161$.

[30] Ma, L., Tan, T., Wang, Y., \& Zhang, D. (2003). Personal identification based on iris texture analysis. Pattern Analysis and Machine Intelligence, IEEE Transactions on, Vol. 25 Issue 12, pp 1519-1533.

[31] Wildes, R. P. (1997). Iris recognition: an emerging biometric technology.Proceedings of the IEEE, Vol. 85 Issue 9, pp 1348-1363.

[32] Prof. Teena Varma, Prof. VidyaChitre,Prof.DiptiPatil, "The Haar Wavelet and The Biorthogonal Wavelet Transforms of an Image", International Journal of Engineering Research and Applications (IJERA) ISSN: 2248-9622 National Conference on Emerging Trends in Engineering \& Technology (VNCET-30 Mar'12) pp 288290

[33] D. de Martin- Roche*, C. Sanchez-Avilat\& R. SanchezReillot Iris Recognition for Biometric Identification 
using DyadicWavelet Transform Zero-Crossing, 272 277. 10.1109/.2001.962844.pp 272-277

[34] CASIA V.3 Iris Image Database Version Three. Available: http://www.cbsr.ia.ac.cn.

[35] Daugman, J. 2006. Probing the Uniqueness and Randomness of Iris Codes: Results from 200 Billion Iris Pair Comparisons. Proceedings of the IEEE. 94: 19271935.

[36] Quinn, G., Grother, P. and Tabassi, E. 2013. Standard Iris Storage Formats. Handbook of Iris Recognition. Springer. 55-66.

[37] Daugman, J. 2004. How Iris Recognition Works. Circuits and Systems for Video Technology, IEEE Transactions on. Vol. 14: pp 21-30.

[38] Daugman, J. 2007. New Methods in Iris Recognition. Systems, Man, and Cybernetics, Part B: Cybernetics, IEEE Transactions on. Vol. 37: pp 1167-1175.

[39] http://www.wisegeek.com/what-is-irisrecognitiontechnology.htm

[40] R.C.Gonzalez and R.E, woods, Digital Image Processing, in J.Houseman (2nd Ed.), Handbook of physiology, 4 (New Jersey: Upper Saddle River, 2002).

[41] Z.He, T.Tan, Z.Sun, and X.Qui, -Towards accurate and fast iris segmentation for iris biometrics, IEEE Trans, On PAMI, Vol. 31, no. 9, pp.1670-1684, Sept. 2009.

[42] A.S Tuama, -Iris Image Segmentation and Recognition,\| International Journal of Computer Science \& Emerging Technologies, IJCSET, Vol.3, no. 2, , April, 2012.

[43] C.Sanchez-Avilla, R. Sanchez- Reillo, D. de MartinRoche, - Iris Based Biometric Recognition using Dyadic Wavelet Transform,\| IEEE AESS Systems Magazine, Oct. 2002.

[44] F. Yan, X. Shao, G. Li, Z. Sun, and Z. Yang, "Edge detection of tank level IR imaging based on the autoadaptive double-threshold canny operator," Intell. Inform. Technology Applicat. Research Assoc., vol. 3, pp. 366 - 370, Dec. 2008.
[45] R. O. Duda and P. E. Hart, "Use of the hough transformation to detect lines and curves in pictures," Commun. the ACM, vol. 15, pp. 11-15, Jan. 1972.

[46] S. Dey, and D. Samanta, J. Daugman, "How iris recognition works," IEEE Trans on Circuits and Systems for Video Technology, vol. 14, no. 1, pp. 21-30, 2004.

[47] Iris Recognition [Online] Available: http://en.wikipedia.org/wiki//ris_recognition

[48] E. Wolff. Antomy of the eye and orbit. 7th edition, H. K. Lewis and Ltd.

[49] I. Daubechies, "Orthonormal Bases of Compactly Supported Wavelets," Comm. Pure Appl. Math., Vol 41, 1988, pp. 906-966.

[50] Haar, Alfréd (1910), "ZurTheorie der orthogonalenFunktionensysteme", MathematischeAnnale n, 69 (3): 331-371

[51] Lee, B.; Tarng, Y. S. (1999). "Application of the discrete wavelet transform to the monitoring of tool failure in end milling using the spindle motor current". International Journal of Advanced Manufacturing Technology. 15 (4): 238-243.

[52] Daugman, J. 2003. The Importance of Being Random: Statistical Principles of Iris Recognition. Pattern Recognition. 36: 279-291.

[53] Monro, D. M. and Zhang, D. 2005. An Effective Human Iris Code with Low Complexity. IEEE International Conference on Image Processing. 3: 277-80.

[54] http://sepwww.stanford.edu/data/media/public/sep/morga n/texturematch/paper_html/node3.html

[55] Li, S. Z. (1998). "Face recognition based on nearest linear combinations". In Proceedings of IEEE Computer Society Conference on Computer Vision and Pattern Recognition, pages 839-844, Santa Barbara, CA.

[56] http://www.scholarpedia.org/article/Nearest_feature_line

[57] Im proceedings Proenca. Hugo and Alexandre, Luis A., -UBIRIS: A noisy iris image database, $\|$ Proceedings of ICIAP 2005- International Conference on Image Analysis and Processing, vol. 1, pp. 970-977. 